\title{
Trends and indicators for quantifying moth abundance and occupancy in Scotland
}

\author{
E. B. Dennis ${ }^{1,2} \cdot$ T. M. Brereton ${ }^{1} \cdot$ B. J. T. Morgan ${ }^{2} \cdot$ R. Fox ${ }^{1} \cdot$ C. R. Shortall ${ }^{3} \cdot$ T. Prescott $^{1} \cdot$ S. Foster $^{4}$
}

Received: 14 January 2019 / Accepted: 31 January 2019 / Published online: 5 April 2019

(c) The Author(s) 2019

\begin{abstract}
Moths form an important part of Scotland's biodiversity and an up-to-date assessment of their status is needed given their value as a diverse and species-rich taxon, with various ecosystem roles, and the known decline of moths within Britain. We use long-term citizen-science data to produce species-level trends and multi-species indicators for moths in Scotland, to assess population (abundance) and distribution (occupancy) changes. Abundance trends for moths in Scotland are produced using Rothamsted Insect Survey count data, and, for the first time, occupancy models are used to estimate occupancy trends for moths in Scotland, using opportunistic records from the National Moth Recording Scheme. Species-level trends are combined to produce abundance and occupancy indicators. The associated uncertainty is estimated using a parametric bootstrap approach, and comparisons are made with alternative published approaches. Overall moth abundance (based on 176 species) in Scotland decreased by $20 \%$ for 1975-2014 and by $46 \%$ for 1990-2014. The occupancy indicator (based on 230 species) showed a $16 \%$ increase for 1990-2014. Alternative methods produced similar indicators and conclusions, suggesting robustness of the results, although rare species may be under-represented in our analyses. Species abundance and occupancy trends were not clearly correlated; in particular species with negative population trends showed varied occupancy responses. Further research into the drivers of moth population changes is required, but increasing occupancy is likely to be driven by a warming summer climate facilitating range expansion, whereas population declines may be driven by reductions in habitat quality, changes in land management practices and warmer, wetter winters.
\end{abstract}

Keywords Abundance $\cdot$ Citizen science $\cdot$ Lepidoptera $\cdot$ Multi-species indicators $\cdot$ National Moth Recording Scheme Occupancy

\section{Introduction}

In recent decades there has been a significant loss of biodiversity in the UK, including Scotland (Hayhow et al. 2016a). Moths form an important component of Scotland's

Electronic supplementary material The online version of this article (https://doi.org/10.1007/s10841-019-00135-z) contains supplementary material, which is available to authorized users.

E. B. Dennis

edennis@butterfly-conservation.org

1 Butterfly Conservation, Wareham, Dorset, UK

2 School of Mathematics, Statistics and Actuarial Science, University of Kent, Canterbury, Kent, UK

3 Rothamsted Insect Survey, Rothamsted Research, Harpenden, UK

4 Scottish Natural Heritage, Inverness, Scotland, UK biodiversity, comprising approximately $3 \%$ of species (1300 of an estimated 46,000 in Scotland, Usher 2002) and are a diverse and species-rich taxon which plays vital roles in ecosystems as food sources for higher trophic levels and in providing pollination services (Merckx et al. 2013; Hahn and Brühl 2016). As they are found in many different habitats and are sensitive to environmental pressures, changes in moth distribution and abundance have potential to be valuable ecological indicators.

Across Great Britain (GB), two-thirds of common and widespread macro-moths have declined over a 40 year period (1968-2007), while others have increased, and the overall abundance of macro-moths decreased by $28 \%$ over the same period (Fox et al. 2013). The potential drivers of these population changes have been highlighted (Fox 2013), including habitat loss, degradation and fragmentation, for example due to agricultural intensification (FuentesMontemayor et al. 2011; Merckx et al. 2012a), changes in 
woodland management (Fuentes-Montemayor et al. 2012; Merckx et al. 2012b), urbanisation (Merckx et al. 2018), chemical pollution, artificial light at night (van Langevelde et al. 2018; Wilson et al. 2018) and climate change (Conrad et al. 2002; Martay et al. 2016).

Moth recording has been well developed in the UK since the 1960s, particularly by the network of traps formed within the Rothamsted Insect Survey (RIS, Conrad et al. 2004), and by volunteer recorders contributing to the National Moth Recording Scheme (NMRS, Fox et al. 2011). However, comprehensive and up-to-date individual or multi-species trends for moths in Scotland have not typically been available, though a composite measure of moth abundance was derived for 1975-2004 using RIS data (Scottish Natural Heritage 2011). This suggested little overall change in common and widespread Scottish moths for the period studied. This status was corroborated by a study of RIS data for 1968-2007 which found no significant change in overall abundance of macro-moths in northern Britain, but a significant decline in southern Britain (Fox et al. 2013).

Until relatively recently, despite the volume of data, records from the NMRS had not been used to estimate robust changes in species distributions, predominantly due to a lack of statistical methods that could account for the sampling bias typical of such opportunistic data (Tingley and Beissinger 2009; Boakes et al. 2010; Hassall and Thompson 2010). Using the Frescalo method (Hill 2012), changes in the frequency of occurrence of macro-moth species in GB showed a range of responses (Fox et al. 2014), but species restricted to northern Britain declined significantly, whereas some moth species that reached their northern range limit in southern Britain increased.

Recent developments in analysis methods bring greater possibilities for measuring changes in both abundance and distribution, in particular through developments in statistical models for seasonal count data (Dennis et al. 2013, 2016a), as well as developments in using opportunistic recording data to derive robust occupancy trends (van Strien et al. 2013; Isaac et al. 2014; Dennis et al. 2017a), which account for variation in the probability of species' detection.

Multi-species indicators are frequently used for assessing the status of groups of species and taxa, for example to assess progress towards national and international biodiversity targets (Tittensor et al. 2014; Burns et al. 2018). However, the methods for producing such indicators are not well developed, in particular when trying to account for missing data and dealing with sources of error. Calculating multispecies indices based on the geometric mean of the specieslevel indices is a popular choice (Buckland et al. 2011), but approaches for accounting for uncertainty vary, from bootstrapping at the site-level (Buckland et al. 2005; Dennis et al. 2017b), bootstrapping across species (Eaton et al. 2015; Burns et al. 2018), or more recently incorporating standard errors of the species-level indices (Soldaat et al. 2017; van Strien et al. 2016).

In this paper various recently developed statistical methods are assessed and tested for producing suitable specieslevel trends as well as multi-species indicators. We undertake an updated analysis of RIS data to assess the abundance status of moths in Scotland, as well as producing distribution trends for moths in Scotland for the first time, using opportunistic records from the NMRS. Multi-species indicators are then produced and compared for abundance and occupancy.

\section{Methods}

\section{Rothamsted insect survey}

The RIS monitors nocturnal moth populations through a UK-wide network of standardised and automated light-traps that are run every night of the year. RIS light-traps tend to capture small (Leinonen et al. 1998) but consistent samples (Taylor and French 1974) which makes the catches reliable and manageable without threatening the moth populations being studied (Williams 1952). The small samples also mean that the traps are best suited for monitoring common and widespread species, for which catches are large enough for statistical analysis (Conrad et al. 2006).

In Scotland, counts have been made at 57 RIS traps and 627 moth species (including 112 micro-moths) have been recorded across the network, over the period 1968-2014. The RIS was established in 1968 but we focus on the period 1975-2014, with 1975 being the start year of the previous moth indicator for Scotland that used RIS data (Scottish Natural Heritage 2009) and 2014 being the last year with available data at the time of analysis. Over the period 1975-2014, moth counts were made at 45 RIS traps in Scotland (Fig. S1) which contributed positive counts for a mean of 10 years (median $=6$ years, range $1-40$ years, Fig. S2). The mean number of traps with positive counts per year was 11 (range 8-18).

Annual indices of abundance (for 1975-2014) were derived from counts from standard RIS light traps (Williams 1948; Woiwod and Harrington 1994). To identify species with sufficient data for estimating RIS trends, we used similar criteria to Conrad et al. (2006) and Scottish Natural Heritage (2011). Firstly, all 185 species included in the previous analysis of moths in Scotland were included (Scottish Natural Heritage 2011), with the exception of five species which were excluded due to potential for misidentification, based on expert opinion (Tom Prescott and Mark Parsons, Butterfly Conservation). A further 56 species with more than 250 individuals captured over the sampling period (1975-2014) were identified for potential inclusion, but then filtered to 28 
species by again excluding species with potential for misidentification. 208 species were therefore identified as having suitable data for trend analysis.

\section{Producing species-level abundance trends}

We fitted a Poisson generalised linear model with year and site factors to annual RIS site indices, using the speedglm package (Enea 2017) in R (R Core Team 2018). Sites which operated in two or more years were included. Scaled predicted year effects were used to form each species' index of relative abundance. Species-level trends in abundance were then estimated using linear regressions to calculate percentage changes for varying time periods, including 1975-2014 (long-term), 1990-2014 (medium-term) and 2005-2014 (short-term). For comparison, abundance trends were also produced using TRIM (Pannekoek and van Strien 2001) fitted with the rtrim package (Bogaart et al. 2018), as well as the spline option of the generalised abundance index (GAI, Dennis et al. 2016a), both of which produced similar conclusions (results not shown here).

Following trend estimation for 208 species (as outlined above), a further 31 species with potentially unreliable (e.g. due to a lack of data) trends were excluded: when the species was counted on at least five sites in fewer than 20 years and the estimated percentage change was $>250 \%$ for either the long or medium-term. One species with a change $>2500 \%$ was also excluded. Thus abundance trends and multi-species indicators are presented for 176 species (including one micro-moth: Diamond-back Moth Plutella xylostella). For the short-term trend (2005-2014), one further species (Cabbage Moth Mamestra brassicae) was excluded as its index was estimated for an insufficient number of years to produce a trend for 2005 onwards.

\section{National moth recording scheme (NMRS)}

Species occurrence records for macro-moths (based on the definition of Fox et al. 2014, defined as Lepidoptera families: Hepialidae, Cossidae, Zygaenidae, Limacodidae, Sesiidae, Lasiocampidae, Saturniidae, Endromidae, Drepanidae, Geometridae, Sphingidae, Notodontidae, Erebidae, Nolidae and Noctuidae) in Scotland were obtained from the NMRS run by Butterfly Conservation (Fox et al. 2011). The records were collated from citizen scientists and verified by expert volunteers during past and ongoing recording for national and local distribution atlases (e.g. Heath and Emmet 1983; Hill et al. 2010). For Scotland, sufficient NMRS data are not available for many species back to 1975 , therefore we chose a base year of 1990 for the occupancy analysis, comparable with the medium term RIS trend.

For initial filtering of species for the period 1990-2014 we used the species list in Fox et al. (2014), where species were excluded (i) that were non-resident species; (ii) that were subject to recent taxonomic revision; (iii) for which recording methodologies changed during the sampling period (e.g. most Sesiidae were excluded because the recent introduction of pheromone lures has greatly improved detection rates); and (iv) that occurred in fewer than 10 grid squares of the Ordnance Survey National Grid in the sampling period.

This left us with 508 species recorded in Scotland from 1990 to 2014, consisting of a total of 919,269 NMRS records. Species believed to have sufficient data to estimate occupancy indices and trends for 1990-2014 were then selected, based on three criteria chosen by expert judgement: being first recorded before 1995 , recorded in at least 15 years of the 25 year period, and having an average of at least 30 records per year. A further five species were excluded due to their potential for misidentification; therefore occupancy analysis was performed for 230 species. The locations of records for these 230 species are shown in Fig. S3.

\section{Producing species-level occupancy trends}

The NMRS data consist of opportunistic records which are not collected using a single protocol, but are instead a collation of both structured and unstructured biological observations. Here we apply a classical occupancy approach based on Dennis et al. (2017a) which analysed similar data for UK butterflies. The approach described below was used to estimate species-level occupancy trends for 1990-2014 (medium-term) and 2005-2014 (short-term).

Occupancy models (MacKenzie et al. 2002) allow for imperfect detection as well as inference on a parameter denoting occupancy probability, but require records of both detection and non-detection at a range of sites. Sites are defined here as a $1 \mathrm{~km} \times 1 \mathrm{~km}$ grid square of the Ordnance Survey National Grid. Hence for opportunistic data, for a given target species, records of all other species are used to provide non-detection records (Kéry et al. 2010). Details of the modelling approach are given in outline here (see Dennis et al. 2017a for more information). Variation in occupancy across sites is described in terms of a set of covariates. For the NMRS data, for any particular species, we model the occupancy probability, $\psi_{i, t}$, for a given site $i$ and year $t$ as a quadratic function of easting and northing on the logistic scale as follows

$\operatorname{logit}\left(\psi_{i, t}\right)=\beta_{0, t}+\beta_{1, t}$ north $_{i}+\beta_{2, t}$ east $_{i}+\beta_{3, t}$ north $_{i}^{2}+\beta_{4, t}$ east $_{i}^{2}$.

Detection probability, for the jth visit to site $\mathrm{i}$ and year $\mathrm{t}$, is described by

$\operatorname{logit}\left(p_{i, t, j}\right)=\alpha_{0, t}+\alpha_{1, t} \log \left(G_{i, t, j}\right)+\alpha_{2, t} V_{j}$,

where $G_{i, t, j}$ is the list length (number of species recorded on visit $j$ to site $i$ in year $t$ ) and $V_{j}$ represents seasonal variation 
by the proportion of records made of the species of interest per week (across all years).

For a given species, an occupancy model was fitted separately to data for each year using the unmarked package (Fiske and Chandler 2011) in R, where maximum likelihood estimates of all of the $\alpha$ and $\beta$ parameters are obtained. For a given species and year $t$, the occupancy index is expressed as the proportion of sites occupied, given by

$I_{t}=\frac{1}{N} \sum_{i=1}^{N} \hat{\psi}_{i, t}$

where $\hat{\psi}_{i, t}$ is estimated for each of $N=97791 \mathrm{~km}$ grid squares in Scotland with at least one record from the NMRS.

In Dennis et al. (2017a), confidence intervals for $I_{t}$ were estimated using a parametric bootstrap approach, but we have found this approach can sometimes underperform in cases with limited data. Hence we instead use the Delta method (Morgan 2009) to approximate the variance of $I_{t}$ as

$\operatorname{var}\left(I_{t}\right)=\left\{\frac{1}{N} \sum_{i=1}^{N}\left(\begin{array}{c}z_{i, t} \\ z_{i, t} \times \text { north }_{i} \\ \vdots \\ z_{i, t} \times \text { east }_{i}^{2}\end{array}\right)\right\} \times \sum_{t} \times\left\{\frac{1}{N} \sum_{i=1}^{N}\left(\begin{array}{c}z_{i, t} \\ z_{i, t} \times \text { north }_{i} \\ \vdots \\ z_{i, t} \times \text { east }_{i}^{2}\end{array}\right)\right\}^{T}$,

where

$$
\begin{aligned}
z_{i, t}= & \frac{e^{y_{i, t}}}{\left(1+e^{y_{i, t}}\right)^{2}}, y_{i, t}=\beta_{0, t}+\beta_{1, t} \text { north }_{i}+\beta_{2, t} \text { east }_{i} \\
& +\beta_{3, t} \text { north }_{i}^{2}+\beta_{4, t} \text { east }_{i}^{2},
\end{aligned}
$$

and $\sum_{t}$ is the estimated variance-covariance matrix for the parameters of the occupancy component of the model for year $t$.

Occupancy trends were estimated by fitting weighted logistic regressions to account for the $(0,1)$ scale of the occupancy indices, using the estimated standard errors of the occupancy index as weightings. Percentage changes for each time period were then estimated from the predicted values from the regressions. For 24 species where the occupancy index started later than 1990 (but not later than 1995), trends for 1990-2014 were estimated by predicting back to 1990 , based on the weighted linear regression.

For comparison, occupancy models were also fitted using a Bayesian approach described in Isaac et al. (2014), implementing code from the Sparta package in R (August et al. 2015).

\section{Producing multi-species indicators}

Multi-species indices were constructed separately for abundance and occupancy by calculating the geometric mean of the species-level indices (Buckland et al. 2011). We used the approach taken in the BRCindicators $\mathrm{R}$ package (August et al. 2017), which accounts for cases where the specieslevel index starts later than the first year, or finishes earlier than the final year. In brief, where a species enters the indicator after the first year, the first year of that species' index is set to the geometric mean of the series for species that are already in the indicator for that year. Furthermore, species that leave the dataset early are held at the last value for the remaining missing years.

Trends were estimated by applying linear regressions to the indicators, and a smoothed indicator was estimated by applying a generalised additive model to the indicator using the $m g c v$ package in $\mathrm{R}$ (Wood 2006), where the smoothing level was chosen automatically using Generalized Cross Validation.

Confidence intervals for the abundance and occupancy indicators were estimated using a parametric bootstrap approach: (i) species-level index values were sampled 1000 times from a normal distribution with estimated mean and standard error of the index for each species and year. The composite indicator was then estimated as above for each of the 1000 samples, and confidence intervals formed by taking the $95 \%$ quantiles. Confidence intervals for the linear trends and smoothed indicator were calculated using the same approach.

For comparison, we explored several alternative approaches for estimating the uncertainty of the abundance indicator: (ii) bootstrapping across species (resampling the species-level indices, see for example Eaton et al. 2015); (iii) bootstrapping from the site-level data (i.e. resampling to produce 500 indices for each species, collated to 500 indicators from which quantiles were estimated, Buckland et al. 2005); and (iv) using the Multi-Species Indicator tool (Soldaat et al. 2017), which can be implemented using the BRCindicators $\mathrm{R}$ package. Bootstrapping site-level data (option iii) accounts for sources of uncertainty from all stages of modelling but is not always practical, whereas option (ii) (bootstrapping the species-level indices) does not account for the standard errors of the estimated abundance indices, and assumes that the set of species is representative of the whole community of interest. The parametric bootstrap (i) and MSI approach (iv) both incorporate the standard errors of the estimated indices via resampling approaches. The MSI approach is based upon Monte Carlo simulation and performs smoothing by LOESS-regression. Soldaat et al. (2017) showed via simulation that bootstrapping by sites produced slightly wider confidence intervals than the MSI approach.

For the occupancy indicator, confidence intervals were produced using approaches (i) and (ii). Furthermore (v), an indicator was also produced from species occupancy indices estimated by the Bayesian occupancy modelling approach, again by the geometric mean, however the confidence intervals were instead formed by taking samples from the posterior densities for the species occupancy indices (Isaac et al. 2018), rather than the resampling approaches tested in the classical case. 
Table 1 Number of species with each abundance trend classification for each time period

\begin{tabular}{lccc}
\hline Trend class & 1975-2014 & 1990-2014 & 2005-2014 \\
\hline Sig. positive & 36 & 5 & 6 \\
No sig. change & 85 & 113 & 153 \\
Sig. negative & 55 & 58 & 16 \\
Total & 176 & 176 & 175 \\
\hline
\end{tabular}

Changes are classified as significant at the $5 \%$ level

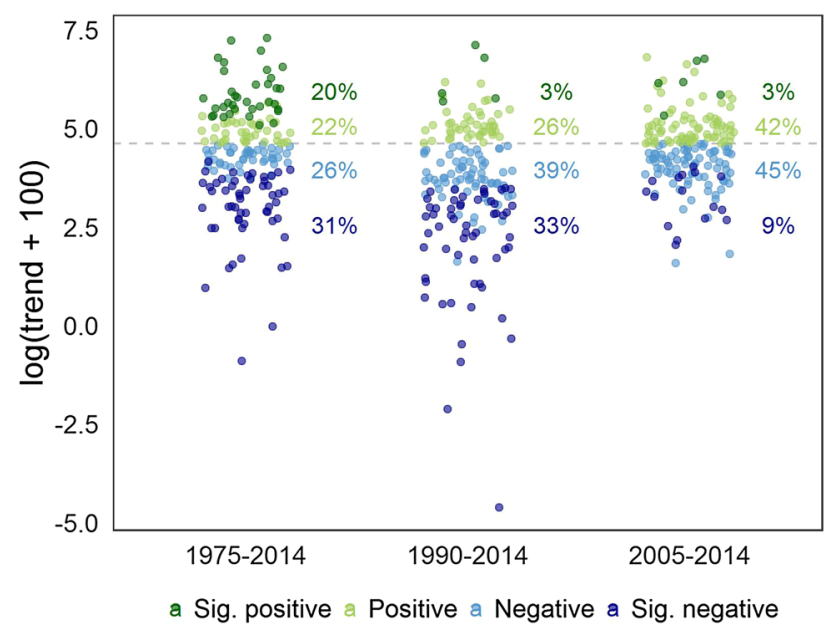

Fig. 1 Summary of 176 species abundance trends $(\log (\operatorname{trend}+100))$ for varying time periods, with indication of the percentage of species belonging to each of four significance categories, where changes are classified as significant at the 5\% level. The dashed horizontal line represents zero change. (Color figure online)

\section{Results}

\section{Abundance trends and indicators}

In each of the three time periods considered, more moth species decreased significantly in abundance than increased (Table 1; Fig. 1). Of the species with significant trend estimates, $60 \%, 92 \%$ and $73 \%$ were negative for the long, medium and short-term respectively. Individual species abundance trends are given in the supplementary material. In each time period assessed, the majority of species showed no significant overall change, and the proportion increased as the time period shortened (48\% of species 1975-2014; 64\% 1990-2014; 87\% 2005-2014), corresponding to reducing sample sizes.

The multi-species indicator (based on 176 species using the parametric bootstrap option i) shows a significant decline in overall abundance for each of the three time periods (Table 2). However, as can be seen from Fig. 2, abundance increased in the early part of the series, but has subsequently declined since the early 1990s. These findings are in keeping with the previous analysis of Scottish RIS data for 1975-2004 (Scottish Natural Heritage 2009), which showed the overall moth trend to be stable ( $+2.6 \%$ based on 185 species).

Similar conclusions can be made from estimating the confidence intervals for the abundance indicator by bootstrapping across species (option ii) or sites (option iii, see Table 2 and Figs. S4 and S5), although in both cases the confidence intervals of the indicator are slightly wider, which influences the estimated significance of the trends. The MSI tool (option iv, Soldaat et al. 2017) produced a similar abundance indicator to the geometric mean approach (Fig. S6), with some overlap in the confidence intervals for the unsmoothed indicators, but there are some differences in the smoothed indicators, which may be influenced by differences in the choice of smoothing between the two methods. Confidence intervals produced by the MSI tool for the smoothed indicator are also considerably narrower than those produced by the parametric bootstrap. The trends estimated by the MSI tool ( $-29 \%$ for $1975-2014$ and $-6.2 \%$ for $2005-2014$ ) are also negative, but do not always fall within the confidence intervals in Table 2.

\section{Occupancy trends and indicators}

For both 1990-2014 and 2005-2014 the majority of species showed no significant overall change in occupancy (Table 3), and in each time period similar numbers of species showed significant positive and negative changes. Ignoring
Table 2 Estimated trends from the abundance indicator for moths in Scotland

\begin{tabular}{lllll}
\hline Time period & \% Change & $95 \%$ CI & \\
\cline { 3 - 5 } & & (i) Parametric bootstrap & (ii) Bootstrap by species & (iii) Bootstrap by sites \\
\hline $1975-2014$ & -20.4 & $(-23.1,-15.1)^{* * *}$ & $(-33.0,-5.5)^{* *}$ & $(-29.1,2.2)$ \\
$1990-2014$ & -45.7 & $(-49.5,-38.9)^{* * *}$ & $(-54.9,-35.2)^{* * *}$ & $(-57.6,-33.5)^{* * *}$ \\
$2005-2014$ & -16.0 & $(-25.4,-7.6)^{* * *}$ & $(-27.6,-2.8)^{*}$ & $(-37.4,-13.1)^{* * *}$ \\
\hline
\end{tabular}

The associated $95 \%$ confidence intervals shown are derived from (i) a parametric bootstrap approach using the standard errors of the species-level indices (ii) bootstrapping the species-level indices (iii) bootstrapping from the site-level data

$* \mathrm{p}<0.05, * * \mathrm{p}<0.01, * * * \mathrm{p}<0.001$ 


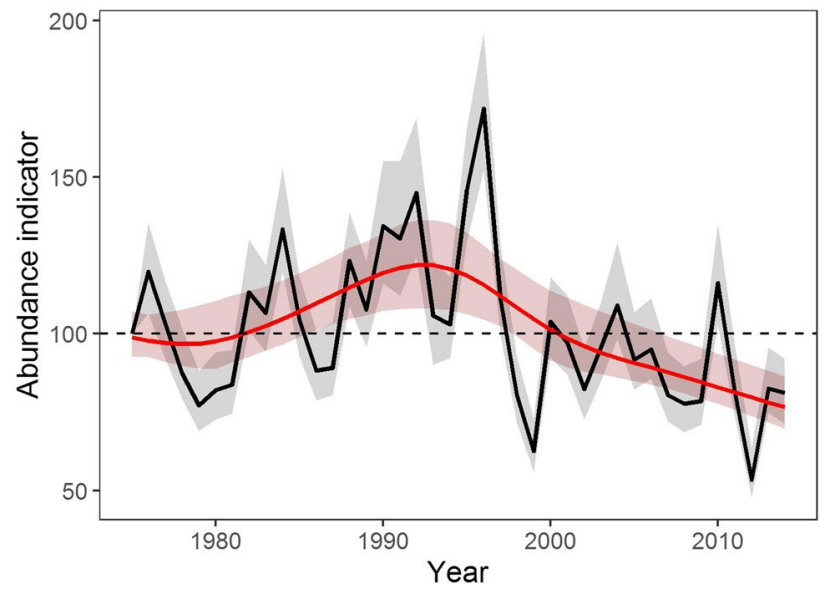

Fig. 2 Moth abundance indicator (black) and smoothed indicator (red), with 95\% confidence intervals derived via the parametric bootstrap approach. The indicator is scaled with respect to a 1975 baseline year (dashed line). (Color figure online)

Table 3 Number of species with each occupancy trend classification for each time period

\begin{tabular}{lcc}
\hline Trend class & $1990-2014$ & $2005-2014$ \\
\hline Sig. positive & 60 & 22 \\
No change & 119 & 188 \\
Sig. negative & 51 & 20 \\
Total & 230 & 230 \\
\hline
\end{tabular}

Changes are classified as significant at the $5 \%$ level

significance, a greater proportion of species showed positive trends (Fig. 3). The full lists of species occupancy trends for 1990-2014 and 2005-2014 are given in the supplementary material.

The multi-species indicator (based on 230 species using the parametric bootstrap option i) shows an increase in occupancy over both periods of assessment (Fig. 4; Table 4). Conclusions from estimating the confidence intervals for the occupancy indicator from the parametric bootstrap (i) or bootstrapping over species (ii) are similar (Table 4 and Fig. S7), although the intervals from the latter are slightly narrower, which is in contrast to the findings for the abundance indicators.

The indicators from the Bayesian (Sparta) and classical occupancy approach show similar increases overall, with some overlap in the confidence intervals for both the unsmoothed and smoothed indicators (Fig. S8), although the intervals from the Bayesian approach are somewhat narrower, and the estimated smoothed line is close to linear. Percentage changes from the Bayesian approach are of a similar order to those in Table 4 (14.1\% for 1990-2014 and $9.0 \%$ for $2005-2014)$.

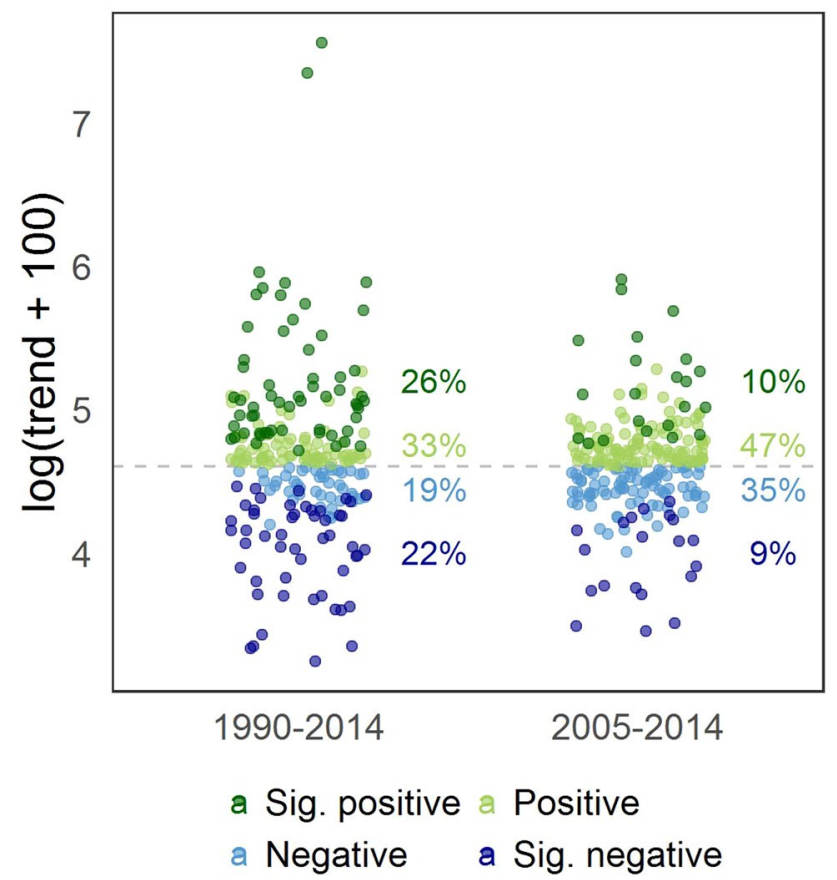

Fig. 3 Summary of 230 species occupancy trends $(\log (\operatorname{trend}+100))$ for two time periods, with indication of the percentage of species belonging to each of four significance categories, where changes are classified as significant at the $5 \%$ level. The dashed horizontal line represents zero change. (Color figure online)

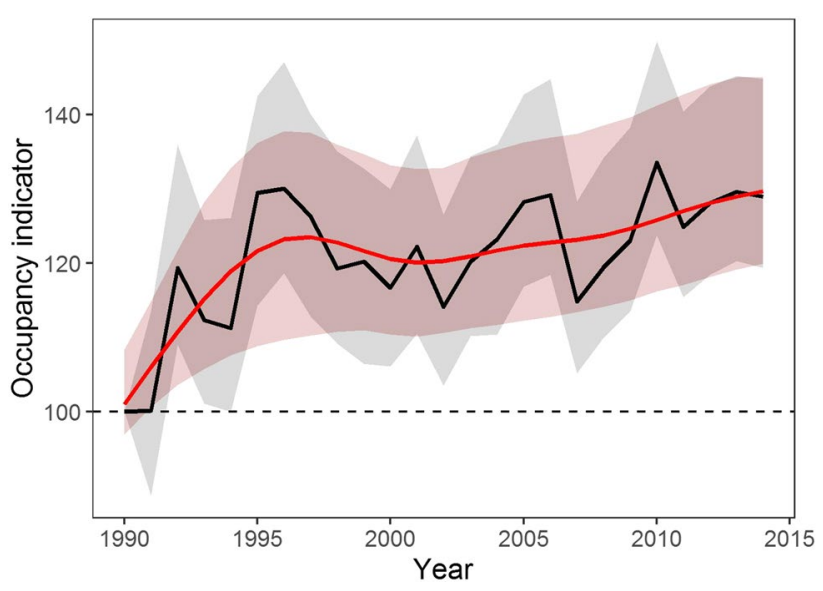

Fig. 4 Moth occupancy indicator (black) and smoothed indicator (red), with 95\% confidence intervals derived via the parametric bootstrap approach. The indicator is scaled with respect to a 1990 baseline year (dashed line). (Color figure online)

\section{Comparing abundance and occupancy}

Trends in both abundance (from RIS) and occupancy (from NMRS) were estimable for 164 moth species for 1990-2014 and 2005-2014. Abundance indicators estimated for the same 164 species were similar to those with the full set of 
Table 4 Estimated trends from the occupancy indicator for moths in Scotland

\begin{tabular}{|c|c|c|c|}
\hline \multirow[t]{2}{*}{ Time period } & \multirow[t]{2}{*}{$\%$ Change } & \multicolumn{2}{|l|}{$95 \% \mathrm{CI}$} \\
\hline & & $\begin{array}{l}\text { (i) Parametric } \\
\text { bootstrap }\end{array}$ & $\begin{array}{l}\text { (ii) Bootstrap by } \\
\text { species }\end{array}$ \\
\hline 1990-2014 & 15.8 & $(12.7,22.4)^{* * *}$ & $(8.1,24.0)^{* * *}$ \\
\hline 2005-2014 & 4.5 & $(3.4,7.5) * * *$ & $(0.4,9.0)^{*}$ \\
\hline
\end{tabular}

The associated $95 \%$ confidence intervals shown are derived from (i) a parametric bootstrap approach using the standard errors of the species-level indices (ii) bootstrapping the species-level indices

$* \mathrm{p}<0.05, * * \mathrm{p}<0.01, * * * \mathrm{p}<0.001$

species, although there were some differences in the estimated occupancy trends, with only a slight overlap in confidence intervals for 1990-2014 (Fig. S9 and Table S1).

Overlooking significance, population trend estimates for 1990-2014 were negative for $70 \%$ of species, compared to negative occupancy trends for $46 \%$ of species (Table 5). Population trend estimates were significant for 58 species, of which most were negative (91\%), compared to significant occupancy trends for 76 species, of which roughly half were negative (51\%).

The abundance and occupancy trend estimates have a small positive correlation (Spearman's rank correlation $\rho=0.17, p=0.029)$. The tendency for more positive occupancy trends (Wilcoxon signed rank test: $V=9739$, $\mathrm{p}<0.001$ ) is shown in Fig. 5, as well as the greater range in magnitude of abundance trends than occupancy trends. Species with negative abundance trend estimates are accompanied by a range of positive and negative occupancy trend estimates. The most extreme negative abundance trends were typically associated with negative or stable occupancy trends. 13 species had significant trends of opposite signs for abundance and occupancy, 12 with significantly negative abundance trends but significantly positive occupancy trends (the greatest difference between trends was for Common Wainscot Mythimna pallens: $-79 \%$ abundance and $+177 \%$ occupancy) and one had a significant positive abundance trend but a significantly negative occupancy trend (Small Phoenix Ecliptopera silaceata).

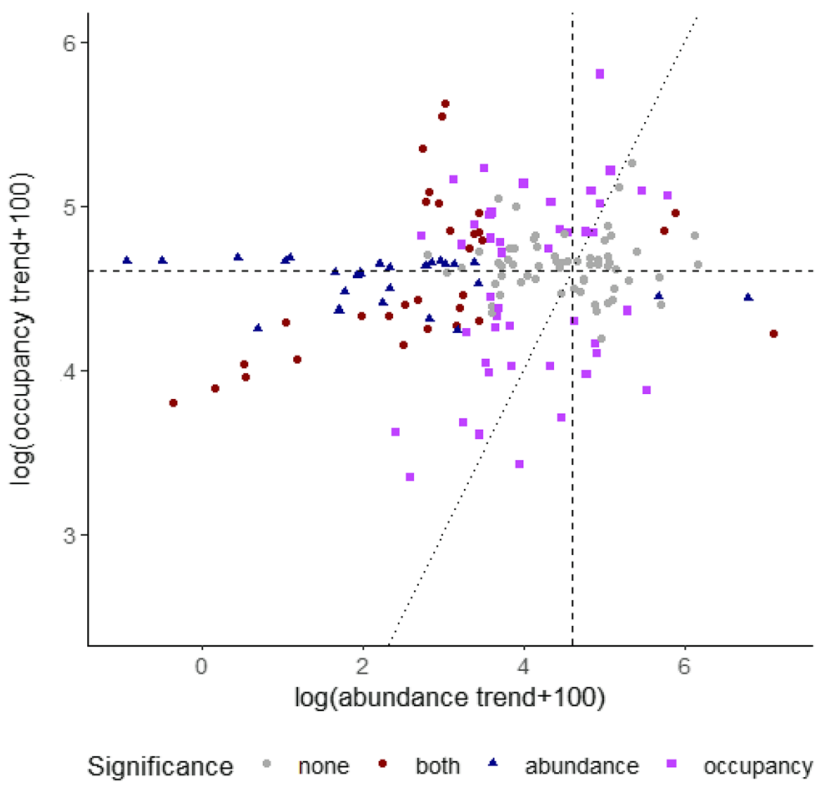

Fig. 5 Comparison of abundance and occupancy trends for 19902014 for 164 species. All trends are on the $\log (\operatorname{trend}+100)$ scale. Dashed lines represent zero change and the dotted line indicates equivalent estimates of abundance and occupancy. Colour of the points classifies the significance of the trends (as significant for neither, both or only abundance or occupancy), where changes are classified as significant at the $5 \%$ level. (Color figure online)

\section{Discussion}

We have produced an updated assessment of moth population trends in Scotland, as well as estimated changes in Scottish moth occupancy for the first time. Abundance and/or occupancy trends were calculable for 242 species ( $43 \%$ of the total), including abundance trends from RIS data for 176 species $(31 \%)$ and occupancy trends from NMRS data for 230 species (41\%). To our knowledge, these analyses provide the first direct comparison of abundance and occupancy trends for a large group of moth species.
Table 5 Comparison of abundance and occupancy trends for 1990-2014 for 164 moths species

\begin{tabular}{|c|c|c|c|c|c|}
\hline & \multicolumn{5}{|l|}{ Occupancy } \\
\hline & Sig. negative & Negative & Positive & Sig. positive & Total \\
\hline \multicolumn{6}{|l|}{ Abundance } \\
\hline Sig. negative & 16 & 11 & 14 & 12 & 53 \\
\hline Negative & 16 & 10 & 21 & 15 & 62 \\
\hline Positive & 6 & 13 & 17 & 8 & 44 \\
\hline Sig. positive & 1 & 2 & 0 & 2 & 5 \\
\hline Total & 39 & 36 & 52 & 37 & 164 \\
\hline
\end{tabular}

Changes are classified as significant at the 5\% level 
Although multi-species indicators provide a useful and effective summary of biodiversity change, the underlying species-level information is unseen and species-level abundance and occupancy trends for moths in Scotland show a mixture of changes. For example, despite the overall decrease in abundance for 1990-2014, estimated population trends were positive for $29 \%$ of species, although only five species had significantly positive trends. Similarly and conversely, despite an overall increase in occupancy, $41 \%$ of species were estimated to have negative occupancy trends for 1990-2014, with 51 species having significantly negative occupancy trends.

A previous analysis of RIS data for Scotland (Scottish Natural Heritage 2009), showed the overall trend to be stable $(+2.6 \%$ for $1975-2004)$. We have updated this indicator using similar species selection criteria and modelling methods, and having evaluated the trend for the same period produced an estimate of a similar magnitude $(+5.1 \%$ for $1975-2004)$.

An estimate of population change for moths across GB as a whole is not currently available for the same time period, but the estimated change over a 40 year period ( $-28 \%$ for $1968-2007$, Fox et al. 2013) showed a decline of a broadly similar order to the 40 year trend of $-20 \%$ estimated here for Scotland, which may indicate that moths in Scotland are declining at a similar rate to those across GB. A decline in overall moth abundance has also been found in the Netherlands (Groenendijk and Ellis 2011).

To our knowledge, moth occupancy trends or other estimates of distribution change are not available for Scotland, from which to make direct comparisons with the trends we estimated. For GB, changes in frequency of occurrence have been estimated for 1970-1990 versus 2000-2010 (Fox et al. 2014), and suggested an overall decrease, but with a variety of changes among the 673 species considered. Species classified as geographically widespread in GB showed an overall decrease, but trends calculated for widespread species for northern GB showed no significant change in frequency of occurrence (Fox et al. 2014). In our occupancy analysis of 230 species in Scotland, all but one species (Pretty Pinion Perizoma blandiata) would be classified as widespread in GB based on the criteria in Fox et al. (2014), and despite the overall increase in occupancy estimated, species-level trends showed a similar mixed pattern of changes to Fox et al. (2014). However we should note the differences in the methods used and the time periods considered.

Species with negative abundance trends showed a mixture of increasing and decreasing occupancy trends. The lack of strong correlation between species abundance and occupancy found for Scottish moths contrasts with previous studies on GB butterflies (Warren et al. 2001; Mair et al. 2014), and cautions against routinely combining both types of information in multi-species indicators (e.g. Hayhow et al. 2016a; van Strien et al. 2016). Van Dyck et al. (2009) also observed some contrasts in species changes in abundance and distribution for butterflies in the Netherlands.

Suitably categorising species-level trends can be difficult, and here we considered changes both in terms of significance but also magnitude, since significance is influenced by sample size (Burns et al. 2018). Species trends have also been plotted explicitly, rather than using popular proportional bar plots, in order to better visualise the variation in species abundance and occupancy trends (Dennis et al. 2018).

Recent work has shown that some British moth populations have undergone large declines and/or range changes, and correlative studies and extrapolation from closely related taxa suggest that agricultural intensification, changes in woodland management and climate change are likely to be major drivers (Fox 2013). The strength of evidence available to assess the drivers of change in moth species is substantially lower than that available for some other animal groups (Burns et al. 2016) and there is a need for further research; hence it is difficult to draw solid conclusions on the drivers of the changes found for Scottish moths in this paper. Climate change is likely to be driving the accelerating northward range expansions recorded for moths in GB (Mason et al. 2015) and therefore the corresponding increases in occupancy, whilst being countered for some species by negative land management and habitat changes, leading to population declines.

Negative environmental changes in Scotland, which may have affected moths, include: commercial afforestation and associated drainage of blanket peat bog; decline in mixed farming; loss/declining quality of semi-natural grassland and moorland through liming, fertilizing, reseeding and overgrazing by livestock; increased extent of built land; loss of broad-leaved and mixed woodland habitat; increased deer grazing pressure and the greater fragmentation and isolation of semi-natural habitats (Scottish Natural Heritage 2009; Hayhow et al. 2016b). It is also possible that some of these changes will have had positive effects for some moth species, especially those that use coniferous trees as larval hostplants. It should be noted that Scotland has a Biodiversity Intactness Index (a measure of the degradation of natural ecosystems) in the lowest fifth of 218 countries analysed (Newbold et al. 2016).

Negative population changes may also be driven by various other influences. For example, warmer wetter winters (which are increasing under climate change) have been shown to negatively impact some moths (Conrad et al. 2002; Hunter et al. 2014) and butterflies (Dennis et al. 2016b; McDermott Long et al. 2017), and there is also growing evidence for negative impacts from light pollution (Macgregor et al. 2015; van Langevelde et al. 2018). 
The species for which it was possible to produce trends, based on the specified selection criteria, were typically common and widespread species, whereas rare and rangerestricted species may be underrepresented due to a lack of sufficient data for the modelling approaches used. The median northing of the ten most northerly records for each of the 230 species was greater in 2005-2014 than 1990-1999 for $70 \%$ of species, suggesting the positive trend in the occupancy indicator is therefore likely to represent range expansion of widespread species which are benefiting from climate warming (Fox et al. 2014). Such changes would be consistent with the general pattern of poleward range expansions documented for other taxa in Britain and globally (Hickling et al. 2006; Chen et al. 2011).

We derived abundance and occupancy indicators, and associated multi-species trends, using published methods that are used in UK and European species biodiversity indices. To our knowledge, the estimation of the uncertainty for the indicators used is novel, but has similarities with the approach of Soldaat et al. (2017). Generally, the outcomes from the various methods considered were similar, and unlikely to lead to different overall conclusions being drawn, providing reassurance that the indicators developed were relatively robust to the methodology used.

In the case of abundance, the parametric bootstrap, which incorporates the estimates of uncertainty associated with the species-level indices, produced slightly narrower confidence intervals than those produced by either bootstrapping from the species or site level. Bootstrapping across species does not account for the uncertainty of the species-level indices, assumes that the species set is representative of all species in the community of interest (Buckland and Johnston 2017; Soldaat et al. 2017), and tends to estimate greater uncertainty (Buckland et al. 2005). Interestingly, the intervals for the occupancy indicator for moths in Scotland were wider from the parametric bootstrap than bootstrapping across species, perhaps because the latter approach does not incorporate the uncertainty associated with the species-level occupancy indices.

Bootstrapping at the site-level may be considered the "gold standard" approach, by accounting for sources of uncertainty from all stages of modelling, but in practice this approach is not always viable either due to the computational demands when working with large volumes of data, or when there is a lack of underlying survey design or when datasets from multiple sources are used (Buckland and Johnston 2017). In this case, it is also possible that the outputs require some caution due to the relatively small sample size for RIS sites in Scotland. The MSI tool (Soldaat et al. 2017) has similarities with the parametric bootstrap proposed in this paper and produced similar unsmoothed indices, although the implemented smoothing resulted in much narrower confidence intervals. Further comparisons, for example via simulation, may provide additional insights on the relative differences and merits of the methods considered here. An additional approach, not considered in this paper, estimates uncertainty based only on the interannual variation of the indicator (Soldaat et al. 2007). Two pitfalls of all the methods considered here, and to our knowledge of the currently available methods, is the potential for poor index estimation in the baseline year (Buckland and Johnston 2017) and the relatively ad hoc approach in which missing data are dealt with, for example when species indices start later than the baseline year.

The classical approach used to calculate the occupancy trends was chosen as it can produce robust trends in a computationally efficient manner. This approach has been shown to produce similar occupancy trends to a Bayesian occupancy approach (Isaac et al. 2014) for a sample of UK butterflies (Dennis et al. 2017a). The two approaches produced similar multi-species trends for Scottish moths but with differences in the indicators themselves, although there were differences in the implementations, for example in the covariates used for the detection probability. The choice of model could warrant further investigation, for example by exploring alternative, less informative priors for the occupancy approach (Outhwaite et al. 2018), or the testing of other occupancy approaches (van Strien et al. 2013), but in practice computationally efficient methods are needed when frequent updates of biodiversity indicators are desired, which can be difficult when fitting models to large-scale citizen science data with potentially limited resources. Although beyond the scope of this analysis, additional spatial covariates could be identified in order to use occupancy models to produce finer-scale analyses of changes in occupancy of moths in Scotland, and potentially assess changes via dynamic maps (Dennis et al. 2017a).

Although the abundance indicator presented suggests a clear signal, some caution is needed given the relatively small number of traps within the RIS network in Scotland, and the tendency for the counts to be small. There is also a risk of bias in the indicators through underrepresenting rare and other specialist moths, as well as day-flying moths, by the use of potentially conservative selection criteria. In particular our selection criteria for the occupancy trends may be considered as strict, but we would favour limiting species inclusion to trends supported by a reasonable quantity of data. However it is possible that alternative selection criteria could be explored. The increasing occupancy trends presented here are likely being driven in part by a warming climate facilitating range expansion, as well as possible other drivers such as changes in woodland management. However, the benefits brought about by the warming summer climate, are potentially being cancelled out by warmer and wetter winters and other environmental changes, leading to population declines. 
The trends and indicators produced here suggest a need for further research to better understand the drivers of recent changes in moth populations in Scotland. The grouped measures of moth abundance and occupancy are comprised of a diverse number of predominantly generalist species. Given that moths are one of the most species-rich wildlife groups in Scotland and have many attributes that make them strong candidates as biodiversity indicators, we believe they could play a highly valuable role in assessing the changing state of biodiversity in Scotland and where data is available, in other countries.

Acknowledgements We thank all the recorders who contribute to the National Moth Recording Scheme. We are grateful to Les Evans-Hill for extraction of the NMRS data. The Rothamsted Insect Survey, a National Capability, is funded by the Biotechnology and Biological Sciences Research Council under the Core Capability Grant BBS/ E/C/000J0200. We are grateful to collaborators and staff who have contributed to the Rothamsted Insect Survey light-trap network since the 1960s. This study was jointly funded by Butterfly Conservation and Scottish Natural Heritage. BJTM was supported by a Leverhulme Emeritus fellowship.

\section{Compliance with ethical standards}

Conflict of interest The authors declare they have no conflict of interest.

Open Access This article is distributed under the terms of the Creative Commons Attribution 4.0 International License (http://creativeco mmons.org/licenses/by/4.0/), which permits unrestricted use, distribution, and reproduction in any medium, provided you give appropriate credit to the original author(s) and the source, provide a link to the Creative Commons license, and indicate if changes were made.

\section{References}

August T, Powney G, Harrower C, Hill M, Isaac N (2015) sparta: Trend Analysis for Unstructured Data. R package version 0.1.30. https:// github.com/BiologicalRecordsCentre/sparta

August T, Powney G, Outhwaite C, Issac N (2017) BRCindicators: Creating biodiversity indicators for species occurrence data. $\mathrm{R}$ package version 1.0. https://github.com/BiologicalRecordsCen tre/BRCindicators

Boakes EH, McGowan PJK, Fuller RA, Chang-qing D, Clark NE, O'Connor K, Mace GM (2010) Distorted views of biodiversity: spatial and temporal bias in species occurrence data. PLoS Biol 8:e1000385

Bogaart P, van der Loo M, Pannekoek J (2018) rtrim: Trends and Indices for Monitoring Data. R package version 2.0.4. https:// CRAN.R-project.org $/$ package $=$ rtrim

Buckland ST, Johnston A (2017) Monitoring the diversity of regions: key principles and possible pitfalls. Biol Cons 214:23-34

Buckland ST, Magurran AE, Green RE, Fewster RM (2005) Monitoring change in biodiversity through composite indices. Phil Trans R Soc B 360:243-254

Buckland ST, Studeny AC, Magurran AE, Illian JB, Newson SE (2011) The geometric mean of relative abundance indices: a biodiversity measure with a difference. Ecosphere 2:1-15
Burns F, Eaton MA, Barlow KE, Beckmann BC et al (2016) Agricultural management and climatic change are the major drivers of biodiversity change in the UK. PLoS ONE 11:e0151595. https ://doi.org/10.1371/journal.pone.0151595

Burns F, Eaton M, Hayhow D, Outhwaite C, Al Fulaij N et al (2018) An assessment of the state of nature in the United Kingdom: a review of findings, methods and impact. Ecol Indic 94:226-236

Chen I, Hill JK, Ohlemüller R, Roy DB, Thomas CD (2011) Rapid range shifts of species associated with high levels of climate warming. Science 333:1024-1026

Conrad KF, Woiwod IP, Perry JN (2002) Long-term decline in abundance and distribution of the garden tiger moth (Arctia caja) in Great Britain. Biol Conserv 106:329-337

Conrad KF, Woiwod IP, Parsons M, Fox R (2004) Long-term population trends in widespread British moths. J Insect Conserv 8:119-136

Conrad KF, Warren M, Fox R, Parsons M, Woiwod IP (2006) Rapid declines of common, widespread British moths provide evidence of an insect biodiversity crisis. Biol Conserv 132:279-291

Dennis EB, Freeman SN, Brereton T, Roy DB (2013) Indexing butterfly abundance whilst accounting for missing counts and variability in seasonal pattern. Methods Ecol Evol 4:637-645

Dennis EB, Morgan BJT, Freeman SN, Brereton TM, Roy DB (2016a) A generalized abundance index for seasonal invertebrates. Biometrics 72(4):305-1314

Dennis EB, Morgan BJT, Freeman SN, Roy DB, Brereton T (2016b) Dynamic models for longitudinal butterfly data. J Agric Biol Environ Stat 21:1-21

Dennis EB, Morgan BJT, Freeman SN, Ridout MS, Brereton TM, Fox R, Powney GD, Roy DB (2017a) Efficient occupancy model-fitting for extensive citizen-science data. PLoS ONE 12:p.e0174433. https://doi.org/10.1371/journal.pone.0174433

Dennis EB, Morgan BJT, Roy DB, Brereton TM (2017b) Urban indicators for UK butterflies. Ecol Ind 76:184-193

Dennis EB, Morgan BJT, Fox R, Roy DB, Brereton T (2018) Functional data analysis of multi-species abundance and occupancy data sets. University of Kent. https://kar.kent.ac.uk/id/eprin $\mathrm{t} / 71360$

Eaton MA et al (2015) The priority species indicator: measuring the trends in threatened species in the UK. Biodiversity 16:108-119

Enea M (2017) speedglm: Fitting linear and generalized linear models to large data sets. R package version 0.3-2. https://CRAN.R-proje ct.org/package $=$ speedglm

Fiske I, Chandler R (2011) unmarked: an R package for fitting hierarchical models of wildlife occurrence and abundance. J Stat Softw 43(10): $1-23$

Fox R (2013) The decline of moths in Great Britain: a review of possible causes. Insect Conserv Divers 6:5-19

Fox R, Randle Z, Hill L, Anders S, Wiffen L, Parsons MS (2011) Moths Count: recording moths for conservation in the UK. J Insect Conserv 15:55-68

Fox R, Parsons MS, Chapman JW, Woiwod IP, Warren MS, Brooks DR (2013) The State of Britain's Larger Moths 2013. Butterfly Conservation and Rothamsted Research, Wareham

Fox R, Oliver TH, Harrower C, Parsons MS, Thomas CD, Roy DB (2014) Long-term changes to the frequency of occurrence of British moths are consistent with opposing and synergistic effects of climate and land-use changes. J Appl Ecol 51:949-957

Fuentes-Montemayor E, Goulson D, Park KJ (2011) The effectiveness of agri-environment schemes for the conservation of farmland moths: assessing the importance of a landscape-scale management approach. J Appl Ecol 48:532-542

Fuentes-Montemayor E, Goulson D, Cavin L, Wallace JM, Park KJ (2012) Factors influencing moth assemblages in woodland fragments on farmland: implications for woodland management and creation schemes. Biol Conserv 153:265-275 
Groenendijk D, Ellis WN (2011) The state of the Dutch larger moth fauna. J Insect Conserv 15:95-101

Hahn M, Brühl CA (2016) The secret pollinators: an overview of moth pollination with a focus on Europe and North America. Anthropod-Plant Interact 10:21-28

Hassall C, Thompson DJ (2010) Accounting for recorder effort in the detection of range shifts from historical data. Methods Ecol Evol $1: 343-350$

Hayhow DB, Burns F, Eaton MA et al (2016a) State of Nature 2016. The State of Nature Partnership

Hayhow DB, Burns F, Eaton MA et al (2016b) State of Nature 2016: Scotland. The State of Nature Partnership

Heath J, Emmet AM (1983) The moths and butterflies of great Britain and Ireland. Harley Books, Colchester

Hickling R, Roy DB, Hill JK, Fox R, Thomas CD (2006) The distributions of a wide range of taxonomic groups are expanding polewards. Glob Change Biol 12:450-455

Hill MO (2012) Local frequency as a key to interpreting species occurrence data when recording effort is not known. Methods Ecol Evol 3:195-205

Hill L, Randle Z, Fox R, Parsons M (2010) Provisional Atlas of the UK's Larger Moths. Butterfly Conservation, Wareham

Hunter MD, Kozlov MV, Itämies J et al (2014) Current temporal trends in moth abundance are counter to predicted effects of climate change in an assemblage of subarctic forest moths. Glob Change Biol 20:1723-1737

Isaac NJB, van Strien AJ, August TA, de Zeeuw MP, Roy DB (2014) Statistics for citizen science: extracting signals of change from noisy ecological data. Methods Ecol Evol 5:1052-1060

Isaac N, Powney G, August T, Outhwaite C, Freeman S (2018) UK Biodiversity Indicators 2018. Technical background document: Deriving indicators from occupancy models. http://jncc.defra.gov. uk/pdf/UKBI2018_TechBG_C4b-D1c_Bayesian.pdf. Accessed Dec 2018

Kéry M, Royle JA, Schmid H, Schaub M, Volet B, Häfliger G, Zbinden N (2010) Site-occupancy distribution modeling to correct population-trend estimates derived from opportunistic observations. Conserv Biol 24:1388-1397

Leinonen R, Söderman G, Itämies J, Rytkönen S, Rutanen I (1998) Intercalibration of different light-traps and bulbs used in moth monitoring in northern Europe. Entomol Fenn 9:37-51

Macgregor CJ, Pocock MJO, Fox R, Evans DM (2015) Pollination by nocturnal Lepidoptera, and the effects of light pollution: a review. Ecol Entomol 40:187-198

MacKenzie DI, Nichols JD, Lachman GB, Droege S, Royle JA, Langtimm CA (2002) Estimating site occupancy rates when detection probabilities are less than one. Ecology 83:2248-2255

Mair L, Hill JK, Fox R, Botham M, Brereton T, Thomas CD (2014) Abundance changes and habitat availability drive species' responses to climate change. Nat Clim Change 4:127-131

Martay B, Monteith DT, Brewer MJ, Brereton T, Shortall CR, PearceHiggins JW (2016) An indicator highlights seasonal variation in the response of Lepidoptera communities to warming. Ecol Ind 68:126-133

Mason SC, Palmer G, Fox R, Fillings S, Hill JK, Thomas CD, Oliver TH (2015) Geographical range margins of many taxonomic groups continue to shift polewards. Biol J Lin Soc 115:586-597

McDermott Long O, Warren R, Price J, Brereton TM, Botham MS, Franco AMA (2017) Sensitivity of UK butterflies to local climatic extremes: which life stage are most at risk? J Anim Ecol $86: 108-116$

Merckx T, Marini L, Feber RE, Macdonald DW (2012a) Hedgerow trees and extended-width field margins enhance macromoth diversity: implications for management. J Appl Ecol 49:1396-1404
Merckx T, Feber RE, Hoare DJ, Parsons MS, Kelly CJ, Bourn NAD, Macdonald DW (2012b) Conserving threatened Lepidoptera: towards an effective woodland management policy in landscapes under intense human land-use. Biol Conserv 149:32-39

Merckx T, Huertas B, Basset Y, Thomas JA (2013) A global perspective on conserving butterflies and moths and their habitats. In: Macdonald DW \& Willis KJ (eds) Key topics in conservation biology 2, Wiley, Oxford, pp. 237-257,

Merckx T, Kaiser A, Van Dyck H (2018) Increased body size along urbanization gradients at both community and intraspecific level in macro-moths. Glob Change Biol 24:3837-3848

Morgan BJT (2009) Applied stochastic modelling, 2nd edn. CRC, Chapman \& Hall, Boca Raton

Newbold T et al (2016) Has land use pushed terrestrial biodiversity beyond the planetary boundary? A global assessment. Science 353:288-291. https://doi.org/10.1126/science.aaf2201

Outhwaite CL, Chandler RE, Powney GD, Collen B, Gregory RD, Isaac, N.J.B (2018) Prior specification in Bayesian occupancy modelling improves analysis of species occurrence data. Ecol Indic 93:333-343

Pannekoek J, van Strien AJ (2001) TRIM 3 manual. TRends and Indices for Monitoring data Research paper no. 0102. Voorburg, The Netherlands: Statistics Netherlands. Available freely at http:// www.ebcc.info

R Core Team (2018) R: a language and environment for statistical computing. R Foundation for Statistical Computing, Vienna

Scottish Natural Heritage (2009) Biodiversity Indicator: S9 Terrestrial insect abundance-moths. https://www.nature.scot/scotlandsindicators-terrestrial-insects. Accessed Aug 2018

Scottish Natural Heritage (2011) Trend Note-Scottish Moths. https:// www.nature.scot/trend-notes-scottish-moths. Accessed Aug 2018

Soldaat L, Visser H, Van Roomen M, Van Strien A (2007) Smoothing and trend detection in waterbird monitoring data using structural time-series analysis and the Kalman filter. J Ornithol 148:351-357

Soldaat LL, Pannekoek J, Verweij RJT, van Turnhout CAM, van Strien AJ (2017) A Monte Carlo method to account for sampling error in multi-species indicator. Ecol Indic 81:340-347

Taylor LR, French RA (1974) Effects of light-trap design and illumination on samples of moths in an English woodland. Bull Entomol Res 63:583-594

Tingley MW, Beissinger SR (2009) Detecting range shifts from historical species occurrences: new perspectives on old data. Trends Ecol Evol 24:625-633

Tittensor DP, Walpole M, Hill SL, Boyce DG, Britten GL et al (2014) A mid-term analysis of progress toward international biodiversity targets. Science 346:241-244

Usher MB (2002) Scotland's biodiversity: trends, changing perceptions and planning for action. In: Usher MB, Mackey EC, Curran J (eds) The state of Scotland's environment and natural heritage. The Stationery Office, Edinburgh

Van Dyck H, van Strien AJ, Maes D, van Swaay CAM (2009) Declines in common, widespread butterflies in a landscape under intense human use. Conserv Biol 23:957-965

van Strien AJ, van Swaay CAM, Termaat T (2013) Opportunistic citizen science data of animal species produce reliable estimates of distribution trends if analysed with occupancy models. J Appl Ecol 50:1450-1458

van Strien AJ, Gmelig Meyling AW, Herder JE et al (2016) Modest recovery of biodiversity in a western European country: the living planet index for the Netherlands. Biol Conserv 200:44-50

van Langevelde F, Braamburg-Annegarn M, Huigens ME et al (2018) Declines in moth populations stress the need for conserving dark nights. Glob Change Biol 24:925-932

Warren MS, Hill JK, Thomas JA et al (2001) Rapid responses of British butterflies to opposing forces of climate and habitat change. Nature 414:65-69 
Williams CB (1948) The Rothamsted light-trap. Proc R Entomol Soc Lond A 23:80-85

Williams CB (1952) Some notes on killing insects for collections and for scientific research. Entomologist 85:271-279

Wilson JF, Baker D, Cheney J, Cook M, Ellis M, Freestone R et al (2018) A role for artificial night-time lighting in long-term changes in populations of 100 widespread macro-moths in UK and Ireland: a citizen-science study. J Insect Conserv 22:189-196

Woiwod IP, Harrington R (1994) Flying in the face of change: the rothamsted insect survey. In: Leigh RA \& Johnston AE (eds)
Long-term experiments in agricultural and ecological sciences, CAB International, London, pp 321-337

Wood SN (2006) Generalized additive models: an introduction with R. Chapman and Hall/CRC, Philadelphia

Publisher's Note Springer Nature remains neutral with regard to jurisdictional claims in published maps and institutional affiliations. 